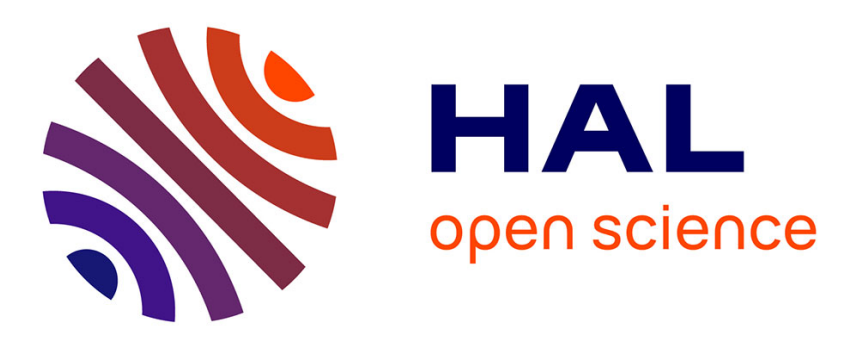

\title{
Accurate deuterium spectroscopy for fundamental studies
}

P. Wcislo, Franck Thibault, M. Zaborowski, S. Wójtewicz, A. Cygan, G. Kowzan, P. Maslowski, J. Komasa, M. Puchalski, K. Pachucki, et al.

\section{- To cite this version:}

P. Wcislo, Franck Thibault, M. Zaborowski, S. Wójtewicz, A. Cygan, et al.. Accurate deuterium spectroscopy for fundamental studies. Journal of Quantitative Spectroscopy and Radiative Transfer, 2018, 213, pp.41-51. 10.1016/j.jqsrt.2018.04.011 . hal-01809053

\section{HAL Id: hal-01809053}

\section{https://hal-univ-rennes1.archives-ouvertes.fr/hal-01809053}

Submitted on 20 Jun 2018

HAL is a multi-disciplinary open access archive for the deposit and dissemination of scientific research documents, whether they are published or not. The documents may come from teaching and research institutions in France or abroad, or from public or private research centers.
L'archive ouverte pluridisciplinaire HAL, est destinée au dépôt et à la diffusion de documents scientifiques de niveau recherche, publiés ou non, émanant des établissements d'enseignement et de recherche français ou étrangers, des laboratoires publics ou privés. 


\section{Highlights}

- weak quadrupole $S(2)$ 2-0 line in self-perturbed D2 is measured

- collisional line-shape effects and energy of this rovibrational transition are calculated

- the velocity-changing collisions are handled with the hardsphere collisional kernel

- the experimental and theoretical pressure broadening and shift are consistent within 5

- we observe 3.4 sigma discrepancy between experimental and theoretical line position 


\title{
Accurate deuterium spectroscopy for fundamental studies
}

\author{
P. Wcisło ${ }^{\text {a }}$, F. Thibault ${ }^{\mathrm{b}}$, M. Zaborowski ${ }^{\mathrm{a}}$, S. Wójtewicz ${ }^{\mathrm{a}}$, A. Cygan ${ }^{\mathrm{a}}$, G. Kowzan $^{\mathrm{a}}$, P. Masłowski $^{\mathrm{a}}$, J. Komasa $^{\mathrm{c}}$, M. Puchalski $^{\mathrm{c}}$, \\ K. Pachucki ${ }^{\mathrm{d}}$, R. Ciuryło ${ }^{\mathrm{a}}$, D. Lisak ${ }^{\mathrm{a}}$

\begin{abstract}
${ }^{a}$ Institute of Physics, Faculty of Physics, Astronomy and Informatics, Nicolaus Copernicus University, Grudziadzka 5, 87-100 Torun, Poland
${ }^{b}$ Institut de Physique de Rennes, UMR CNRS 6251, Université de Rennes 1, Campus de Beaulieu, Bât.11B, F-35042 Rennes, France

${ }^{c}$ Faculty of Chemistry, Adam Mickiewicz University, Umultowska 89b, 61-614 Poznań, Poland

${ }^{d}$ Faculty of Physics, University of Warsaw, Pasteura 5, 02-093 Warsaw, Poland
\end{abstract}

\begin{abstract}
We present an accurate measurement of the weak quadrupole $\mathrm{S}(2)$ 2-0 line in self-perturbed $\mathrm{D}_{2}$ and theoretical ab initio calculations of both collisional line-shape effects and energy of this rovibrational transition. The spectra were collected at the 247 - 984 Torr pressure range with a frequency-stabilized cavity ring-down spectrometer linked to an optical frequency comb (OFC) referenced to a primary time standard. Our line-shape modeling employed quantum calculations of molecular scattering (the pressure broadening and shift and their speed dependencies were calculated, while the complex frequency of optical velocity-changing collisions was fitted to experimental spectra). The velocity-changing collisions are handled with the hard-sphere collisional kernel. The experimental and theoretical pressure broadening and shift are consistent within 5\% and 27\%, respectively (the discrepancy for shift is $8 \%$ when referred not to the speed averaged value, which is close to zero, but to the range of variability of the speed-dependent shift). We use our high pressure measurement to determine the energy, $v_{0}$, of the $\mathrm{S}(2)$ 2-0 transition. The $a b$ initio line-shape calculations allowed us to mitigate the expected collisional systematics reaching the $410 \mathrm{kHz}$ accuracy of $v_{0}$. We report theoretical determination of $v_{0}$ taking into account relativistic and QED corrections up to $\alpha^{5}$. Our estimation of the accuracy of the theoretical $v_{0}$ is $1.3 \mathrm{MHz}$. We observe $3.4 \sigma$ discrepancy between experimental and theoretical $v_{0}$.
\end{abstract}

Keywords: Molecular deuterium, Molecular collisions, Spectral line shapes, Absolute frequency measurements, Cavity ring-down spectroscopy, Quantum electrodynamics in molecules

\section{Introduction}

Molecular hydrogen in its ground electronic state, the simplest neutral chemically bound system, constitutes the most suitable platform for testing quantum electrodynamics (QED) for molecules and for searching for new physics beyond the standard model [1], such as new forces [2] or extra dimensions [3]. However, direct experimental studies on the rovibrational structure of $\mathrm{H}_{2}$ are difficult to perform because of the absence of strong dipole coupling between the levels. Typical Doppler-free saturation spectroscopy is not applicable to weak quadrupole molecular transitions. Two independent experimental strategies have been advanced to reach the megahertz level of accuracy in the determination of the frequencies of rovibrational lines. The first one [4-8] is based on Doppler-free two-photon spectroscopy of electronic transitions in molecular beams. Two different vibrational levels in the ground electronic state were coupled to the same excited electronic level. This allowed the energy difference between the $(v=1, j=0)$ and $(v=0, j=0)$ ground electronic states to be determined with an accuracy of $2.8 \mathrm{MHz}$ [7]. This approach has great potential for improvements because of recent progress in Ramsey-comb spectroscopic techniques [9]. The second strategy operates in the Doppler-limited regime. It takes advantage of ultra-high finesse cavities to directly measure

Email address: piotr.wcislo@fizyka.umk.pl (P. Wcisło) the weak quadrupole lines and has already achieved the kilohertz level of accuracy [10-12] typical for Doppler-free techniques. However, in the case of molecular hydrogen, the collisional line-shape effects are very pronounced and untypical [13-15]. As a result, the line position does not scale linearly with pressure [16]; this phenomenon usually is not taken into account [17]. To avoid such systematic errors, Mondelain et al. [18] measured the $\mathrm{D}_{2} \mathrm{~S}(2)$ 2-0 line at very low pressure, where collisions are negligible, reaching an accuracy of $0.50 \mathrm{MHz}$.

In this article, we present experimental and theoretical studies on the shape of the $D_{2} S(2)$ 2-0 line, which allowed us to bring the previous measurements of the line position into the high-pressure range and reach (despite a twenty times shorter effective optical path) the same sub-megahertz level of accuracy as the Doppler-regime measurements [18]. The spectra were recorded with a frequency-stabilized cavity ring-down spectrometer linked to an optical frequency comb (OFC) referenced to a primary time standard. The experiment was carried out at room temperature and pressures varying from 247 to 984 Torr. In contrast to most of the works devoted to the analysis of the shapes of experimental molecular lines, where simple phenomenological models (such as hard- $[19,20]$ or soft-collision [21] models of the velocity-changing collision and quadratic or hypergeometric approximations of the speed dependence) are applied, we employ an approach originating from first principles 
$[14,15,22,23]$. We performed fully quantum calculations of molecular scattering to obtain the pressure broadening and shift and their speed dependencies. The complex frequency of optical velocity-changing collisions was fitted to experimental spectra. The velocity-changing collisions are handled with a collisional kernel that originates from the hard-sphere approximation of the potential energy surface [14] (the resulting profile is called speed-dependent billiard-ball profile [24] with ab initio speed dependence). Our experimental and theoretical determinations of the pressure broadening are consistent within 5\%. The relative discrepancy for the pressure shift is $27 \%$. This value is, however, misleading because for this system the shift is large and strongly depends on absorber speed, but its speed-averaged value is close to zero. Therefore to provide better measure of the relative discrepancy one should refer the difference not to the speed-averaged value but to the range of the shift variability and then the relative discrepancy equals to $8 \%$. The real part of the fitted frequency of the optical velocity-changing collisions is consistent with the frequency of the velocity-changing collisions calculated from kinetic theory. We use our measurement to determine the energy, $v_{0}$, of the $\mathrm{S}(2) 2-0$ transition. The $a b$ initio line-shape calculations allowed us to mitigate the expected collisional systematics (the line asymmetry originating from speed dependence of collisional shift) reaching the accuracy of $401 \mathrm{kHz}$.

We report theoretical determination of $v_{0}$ taking into account relativistic and QED corrections up to $\alpha^{5}$. Our estimation of the accuracy of the theoretical $v_{0}$ is $1.3 \mathrm{MHz}$. We observe $3.4 \sigma$ discrepancy between experimental and theoretical $v_{0}$. The reason for this discrepancy is not known.

\section{Ab initio line-shape modeling}

\subsection{Velocity distribution of an optical coherence at the station-} ary state

The shapes of molecular lines can be modeled by determining the velocity distribution of an optical coherence associated with the considered transition from the transport-relaxation equation $[22,23,25-27]$

$$
f_{m}(\vec{v})=-i\left(\omega-\omega_{0}-\vec{k} \cdot \vec{v}\right) f(\omega, \vec{v})-\hat{S} f(\omega, \vec{v}),
$$

where $\omega$ and $\omega_{0}$ are the angular frequency of the electromagnetic radiation and the unperturbed angular frequency of the molecular transition. In the experimental data analysis, see Sec. 3, we use $v=\omega /(2 \pi)$ and $v_{0}=\omega_{0} /(2 \pi)$. The $\vec{k} \vec{v}$ term is the Doppler shift, where $\vec{v}$ is the velocity of an active molecule and $\vec{k}$ is the wave vector. $f_{m}(\vec{v})=\left(\sqrt{\pi} v_{m}\right)^{-3} e^{\left(-v / v_{m}\right)^{2}}$ is the Maxwell velocity distribution, where $v_{m}=\sqrt{2 k_{B} T / m_{1}}$ is the most probable speed. $k_{B}, T$ and $m_{1}$ are the Boltzmann constant, temperature and mass of the active molecule, respectively. $\hat{S}$ is the collision operator describing the relaxation and dephasing of the optical coherence as well as its flow between different velocity classes. Since the $\mathrm{D}_{2}$ rovibrational lines are very well separated (the rotational constant is exceptionally large, about $30 \mathrm{~cm}^{-1}$ ), we restrict our discussion to the case of isolated lines, i.e., we neglect line mixing [28]. Having $f(\omega, \vec{v})$, which is a scalar function proportional to the velocity distribution of the optical coherence, the line-shape function can be calculated as

$$
I(\omega)=\frac{1}{\pi} \operatorname{Re} \int f(\omega, \vec{v}) d^{3} \vec{v} .
$$

The Maxwell distribution is factored out from the solution of Eq. (1), $f(\omega, \vec{v})=f_{m}(\vec{v}) h(\omega, \vec{v})$; hence, the equation takes the following form

$$
1=-i\left(\omega-\omega_{0}-\vec{k} \vec{v}\right) h(\omega, \vec{v})-\hat{S}^{f} h(\omega, \vec{v}),
$$

where $\hat{S}^{f}$ is defined as $\hat{S} f_{m}(\vec{v}) h(\omega, \vec{v})=f_{m}(\vec{v}) \hat{S}^{f} h(\omega, \vec{v})$. In the general case, Eq. (3) can be solved by decomposing functions and operators in some basis and hence converting Eq. (3), which is a multidimensional integral Boltzmann-like equation, into a set of algebraic equations. If we introduce an orthonormal basis, $\left\{\phi_{s}(\vec{v})\right\}$, and define a scalar product, $\left\langle\phi_{s^{\prime}} \mid \phi_{s}\right\rangle=$ $\int d^{3} \vec{v} f_{m}(\vec{v}) \phi_{s^{\prime}}(\vec{v}) \phi_{s}(\vec{v})$, then Eq. (3) can be written as [27]

$$
\underline{\mathbf{b}}=\left(-i\left(\omega-\omega_{0}\right) \mathbf{1}+i \mathbf{K}-\mathbf{S}^{f}\right) \underline{\mathbf{c}}(\omega),
$$

where column $\mathbf{c}(\omega)$ is $c_{s}(\omega)=\left\langle h(\omega, \vec{v}) \mid \phi_{s}(\vec{v})\right\rangle$, matrix $\mathbf{K}$ is $[\mathbf{K}]_{s^{\prime}, s}=\left\langle\phi_{s^{\prime}}|\overrightarrow{\vec{k}} \vec{v}| \phi_{s}\right\rangle, \mathbf{1}$ is the identity matrix, $\underline{\mathbf{b}}$ is a column defined as $[\underline{\mathbf{b}}]_{s}=\delta_{s, 0}\left(\delta_{s^{\prime}, s}\right.$ is the Kronecker delta $)$, and matrix $\mathbf{S}^{f}$ is defined as $\left[\mathbf{S}^{f}\right]_{s^{\prime}, s}=\left\langle\phi_{s^{\prime}}\left|\hat{S}^{f}\right| \phi_{s}\right\rangle$. In practice, the infinite system of coupled linear equations (4) is reduced to a system of at most a few thousand equations. If the first element of the basis is a constant function, i.e., $\phi_{0}(\vec{v})=1$, then the line-shape function, Eq. (2), can be written as [27]

$$
I(\omega)=\frac{1}{\pi} \operatorname{Re}\left\langle\phi_{0}(\vec{v}) \mid h(\omega, \vec{v})\right\rangle=\frac{1}{\pi} \operatorname{Re}\left[c_{0}(\omega)\right] .
$$

In this work, we use the Burnett functions basis defined as

$$
\begin{aligned}
\psi_{n l m}\left(\vec{v} / v_{m}\right)= & \sqrt{2 \pi^{3 / 2} n ! / \Gamma(n+l+3 / 2)} \\
& \cdot\left(v / v_{m}\right)^{l} L_{n}^{l+1 / 2}\left(\left(v / v_{m}\right)^{2}\right) Y_{l m}(\theta, \phi)
\end{aligned}
$$

where $L_{n}^{l+1 / 2}\left(\left(v / v_{m}\right)^{2}\right)$ is an associated Laguerre polynomial, $Y_{l m}(\theta, \phi)$ is the usual spherical harmonic function, and $\{v, \theta, \phi\}$ are spherical coordinates of $\vec{v}$.

\subsection{The collisional operator $\hat{S}^{f}$}

The collisional operator, $\hat{S}^{f}$, can be arbitrarily divided into two components [29]:

$$
\hat{S}^{f}=\hat{S}_{\mathrm{D}}^{f}+\hat{S}_{\mathrm{VCD}}^{f}
$$

where the $\hat{S}_{\mathrm{D}}^{f}$ operator describes the relaxation of the internal motion of the molecule, i.e., the damping and dephasing of the optical coherence. The $\hat{S}_{\mathrm{VCD}}^{f}$ operator describes the remaining part of $\hat{S}^{f}$, i.e., the flows of the optical coherence between different velocity classes affected by its simultaneous damping and dephasing.

In the idealized case when the changes in the molecule velocity are completely independent from (uncorrelated with) 


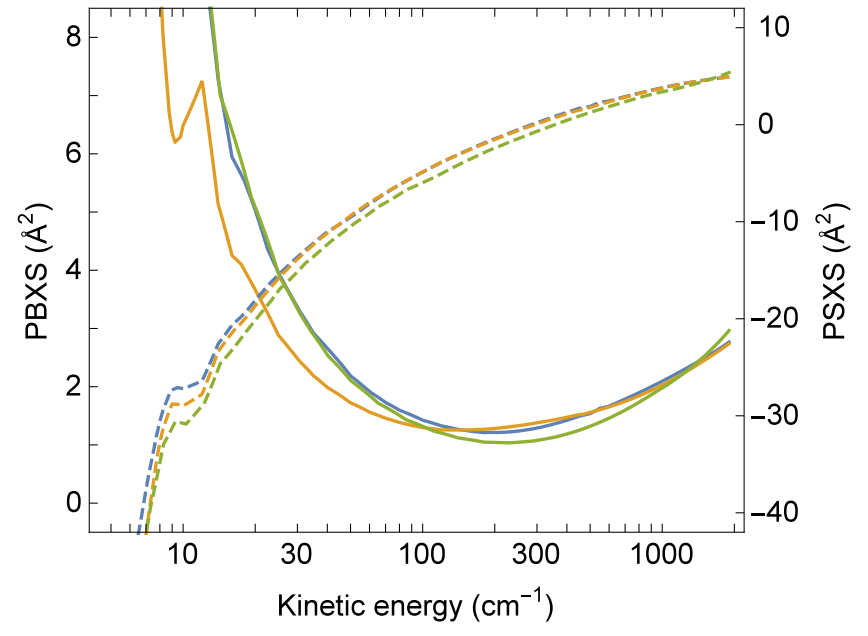

Figure 1: Generalized spectroscopic cross sections for the $S(2)$ 2-0 transition in $\mathrm{D}_{2}$ as a function of collisional kinetic energy. Pressure-broadening cross sections (PBXS) and pressure-shift cross sections (PSXS) are shown as solid and dashed lines, respectively. For readability, we show the results for three rotational states of perturbing molecules (out of six used in our calculations, i.e., $j_{2}=0$ to 5 ) The blue, yellow and green colors correspond to $j_{2}=0,1$ and 4 , respectively.

the damping and dephasing, then $\hat{S}_{\mathrm{VCD}}^{f}$ is equal to the usual Boltzmann collisional operator $\hat{S}_{\mathrm{VC}}^{f}[25,30,31]$

$$
\begin{aligned}
& \hat{S}_{\mathrm{VC}}^{f} h(\omega, \vec{v})=n_{2} \int d^{3} \vec{v}_{2} \int d \tilde{\Omega}\left(\frac{d \sigma}{d \tilde{\Omega}}\right)\left|\vec{v}-\vec{v}_{2}\right| \cdot \\
& \cdot f_{m_{2}}\left(\vec{v}_{2}\right)\left(h\left(\omega, \vec{v}^{\prime}\right)-h(\omega, \vec{v})\right)
\end{aligned}
$$

where $n_{2}$ is the concentration of perturbers, $\tilde{\Omega}$ is the scattering solid angle, $d \sigma / d \tilde{\Omega}$ is the differential elastic cross section, $\vec{v}^{\prime}$ is the velocity of an absorber after collision, $\vec{v}_{2}$ is the velocity of a perturber, and $f_{m_{2}}\left(\vec{v}_{2}\right)$ is the velocity distribution of the perturbers. The $\hat{S}_{\mathrm{VC}}^{f}$ operator can also be expressed in terms of the collision kernel $f\left(\vec{v} \leftarrow \vec{v}^{\prime}\right)$ describing probability rates of velocity changes from $\vec{v}^{\prime}$ to $\vec{v}$ :

$$
\begin{aligned}
& \hat{S}_{\mathrm{VC}}^{f} h(\omega, \vec{v})= \\
& \int d^{3} \vec{v}^{\prime}\left[\frac{f_{m}\left(\vec{v}^{\prime}\right)}{f_{m}(\vec{v})} f\left(\vec{v} \leftarrow \vec{v}^{\prime}\right) h\left(\omega, \vec{v}^{\prime}\right)-f\left(\vec{v}^{\prime} \leftarrow \vec{v}\right) h(\omega, \vec{v})\right] .
\end{aligned}
$$

To quantify the frequency of the velocity changes, we also define a normalized form, $\hat{M}$, of this operator

$$
\hat{S}_{\mathrm{VC}}^{f}=v_{\mathrm{VC}} \hat{M}^{f}
$$

where $v_{\mathrm{VC}}$ is the effective frequency of the velocity-changing collisions, which can be calculated as

$$
v_{\mathrm{VC}}=\frac{v_{m}^{2}}{2 D}
$$

where $D$ is the mass diffusion coefficient.

Equations (8) to (11) are valid when there is no correlation between the velocity-changing and damping/dephasing collisions, which is not the case for realistic systems. The correlations are accounted for in the generalized Hess method (GHM) [3234]. Within the GHM framework, the $\hat{S}_{\mathrm{D}}^{f}$ operator is simply a complex number, $-\left(\Gamma_{0}+i \Delta_{0}\right)$, where $\Gamma_{0}$ and $\Delta_{0}$ are speedaveraged collisional broadening and shift, respectively. The GHM allows the $\hat{S}_{\mathrm{VCD}}^{f}$ operator to be identified with $v_{\mathrm{opt}} \hat{M}_{\mathrm{HC}}^{f}$, where $\hat{M}_{\mathrm{HC}}^{f}$ is the normalized Boltzmann collisional operator, see Eqs. (9) and (10), for the case of the simple hard-collision kernel

$$
f\left(\vec{v} \leftarrow \vec{v}^{\prime}\right)=v_{\text {opt }} f_{m}(\vec{v}) .
$$

Therefore, the GHM allows us to write the full collision operator $\hat{S}^{f}$ as

$$
\hat{S}^{f}=-\Gamma_{0}-i \Delta_{0}+\nu_{\mathrm{opt}} \hat{M}_{\mathrm{HC}}^{f}
$$

The $v_{\text {opt }}$ parameter is called frequency of optical velocitychanging collisions; it has to be emphasized that it is a complex number. The real part of it, $v_{\mathrm{opt}}^{r}$, is smaller than $v_{\mathrm{VC}}$. The reason for this is that some part of the optical coherence, which could flow to different velocity classes, is damped or dephased. The nonzero imaginary part, $v_{\mathrm{opt}}^{i}$, results from the fact that during the velocity-changing collision, the phase of the optical coherence is changed as well. Within GHM, the $\Gamma_{0}, \Delta_{0}$ and $v_{\mathrm{opt}}$ parameters are calculated from first principles as

$$
\begin{gathered}
\Gamma_{0}+i \Delta_{0}=\frac{1}{2 \pi c} \frac{p}{k_{B} T} \sum_{j_{2}} p_{j_{2}} \omega_{0}^{00}\left(q, j_{2}\right), \\
v_{\mathrm{opt}}=\frac{1}{2 \pi c} \frac{p}{k_{B} T} M_{2} \sum_{j_{2}} p_{j_{2}}\left[\frac{2}{3} \omega_{1}^{11}\left(q, j_{2}\right)-\omega_{0}^{00}\left(q, j_{2}\right)\right],
\end{gathered}
$$

where the collision integral $\omega_{\lambda}^{s, s^{\prime}}\left(q, j_{2}\right)$ is given by the following expression $[35,36]$ :

$$
\begin{aligned}
& \omega_{\lambda}^{s, s^{\prime}}\left(q, j_{2}\right)= \\
& \left\langle v_{r}\right\rangle \int_{0}^{\infty} d x x^{\left(s+s^{\prime}+2\right) / 2} e^{-x} \sigma_{\lambda}^{q}\left(v_{i} j_{i} v_{f} j_{f} j_{2} ; E_{k i n}=x k_{B} T\right),
\end{aligned}
$$

where $\left\langle v_{r}\right\rangle=\sqrt{8 k_{B} T / \pi \mu}$ and $\mu$ is the reduced mass of the colliding partners. $q$ is the tensor rank of the spectral transition operator (for the quadrupole line considered here $q=2$ ). $T$, $k_{B}$ and $p$ are the temperature, Boltzmann constant and pressure, respectively. The $1 /(2 \pi)$ factor appears in Eqs. (14) and (15) because the horizontal axis of the spectrum is expressed in terms of frequency, $v$, and not angular frequency, $\omega$, as it is in Eq. (1). The $1 / c$ factor converts the frequency unit into wavenumbers. $M_{2}=m_{2} /\left(m_{1}+m_{2}\right)$, where $m_{1}$ and $m_{2}$ are the masses of the active and perturbing molecules. In the self-perturbed case, as it is in this paper, $M_{2}=1 / 2 . p_{j_{2}}$ is the population of the $j_{2}$ state of the perturber molecule at temperature $T\left(j_{2}\right.$ is the rotational quantum number of the perturbing molecule before the collision)

$$
p_{j_{2}}=w_{j_{2}}\left(2 j_{2}+1\right) e^{-E_{j_{2}} /\left(k_{B} T\right)} / Z(T),
$$

where $Z(T)$ is the corresponding partition function

$$
Z(T)=\sum_{j_{2}} w_{j_{2}}\left(2 j_{2}+1\right) e^{-E_{j_{2}} /\left(k_{B} T\right)} .
$$


The $w_{j_{2}}$ weight arises from the symmetry condition for the total wavefunction of the $\mathrm{D}_{2}$ molecule (the deuterium atom is a boson) and the degeneracy in the total nuclear spin $I$. For even $j_{2}$, the total nuclear spin can be $I=0$ or 2 ; hence, $w_{j_{2}}=6$ for orthostates. For odd $j_{2}$, the total nuclear spin is $I=1$; hence, $w_{j_{2}}=3$ for para states. $E_{j_{2}}$ is the energy of the $j_{2}$ state $\left(E_{j_{2}=0}=0\right)$. $\sigma_{\lambda}^{q}\left(v_{i} j_{i} v_{f} j_{f} j_{2} ; E_{k i n}\right)$ is a generalized spectroscopic cross section, where $v_{i} j_{i}$ and $v_{f} j_{f}$ are vibrational and rotational quantum numbers for the initial and final spectroscopic states (for the 2-0 $\mathrm{S}(2)$ line $v_{i}=0, j_{i}=2, v_{f}=2$ and $\left.j_{f}=4\right) . E_{k i n}$ is the initial relative center-of-mass kinetic energy. The $\sigma_{\lambda}^{q}\left(v_{i} j_{i} v_{f} j_{f} j_{2} ; E_{k i n}\right)$ cross sections are calculated from the scattering $\mathrm{S}$-matrices. The explicit expression for $\sigma_{\lambda}^{q}\left(v_{i} j_{i} v_{f} j_{f} j_{2} ; E_{k i n}\right)$ is given in Refs. [34] and [35]; see Eq. (34) in Ref. [34] and Eq. (2) in Ref. [35]. Note that our $\sigma_{\lambda}^{q}\left(v_{i} j_{i} v_{f} j_{f} j_{2} ; E_{k i n}\right)$ has dimensions of area, while that in Ref. [34] is dimensionless. The conversion factor is $\pi / k^{2}$, where $k^{2}=2 \mu E_{k i n} / \hbar^{2}$. Note that $\sigma_{0}^{q}$ is a standard generalized spectroscopic cross section [37-39]. In Fig. 1, we present illustrative examples of calculated values of $\sigma_{0}^{2}\left(v_{i}=0, j_{i}=2, v_{f}=2, j_{f}=4, j_{2} ; E_{k i n}\right)$ for $j_{2}=0,1$ and 4 . We refer to the real and imaginary parts of $\sigma_{0}^{q}$ as the pressurebroadening cross-section (PBXS) and the pressure-shifting crosssection (PSXS), respectively. We truncated the summation over $j_{2}$ at 5 . At the considered temperature, $T=294.9 \mathrm{~K}$, the cumulated population of the $j_{2}>5$ states is $0.6 \%$ of the total population (the $j_{2}$ dependence is weak, and therefore, the corresponding error of the cross sections will be much smaller). Dynamical calculations were performed on Hinde's PES [40], and the S-matrices were determined by solving the close-coupling equations using the MOLSCAT code [41].

The hard-collision model is insufficient for a proper description of the velocity-changing collisions in the modeling of the shapes of molecular resonances, in particular, the shapes of molecular hydrogen lines $[15,16,42-44]$. The phenomenological hard-collision kernel, Eq. (12), obeys the basic thermodynamic requirements (at equilibrium, the velocity distribution converges to the Maxwellian distribution and the detailed balance relation is satisfied), but it is not based on the interaction potential. The physical meaning of the hard-collision kernel, Eq. (12), is that the velocity of the molecule is completely thermalized after each collision regardless of the pre-collisional velocity. The line-shape effects are physically better described when the $\mathrm{VC}$ collisions are obtained from the interaction potential of the colliding pair [14]. In panel(b) in Fig. 2, we show the isotropic part of the $D_{2}-D_{2}$ potential (black line) and its hard-sphere approximation (blue line). As a reference, we also show the Maxwell-Boltzmann collision energy distribution in panel (a). It has been demonstrated, by a direct comparison with the $a b$ initio classical molecular dynamics simulations [42], that the hard-sphere approximation very well describes the dynamics of the velocity-changing collisions for the case of self-perturbed hydrogen [14]. In contrast to the HC kernel, the hard-sphere kernel (also called the billiard-ball kernel, and hence, we refer to it as the BB kernel) depends on the velocity before the collision and the scattering angle $\theta[14,45]$

$$
\begin{aligned}
& f_{\mathrm{BB}}\left(\vec{v} \leftarrow \vec{v}^{\prime}\right)=v^{(0)} \frac{1}{v_{m}^{2}} \frac{3}{32 \pi} \frac{\sqrt{1+\alpha}(1+\alpha)^{2}}{\alpha^{2}} \frac{1}{\sqrt{v^{2}-2 v v^{\prime} \cos \theta+v^{\prime 2}}} \times \\
& \quad \times \exp \left(-\frac{(1-\alpha)^{2}}{4 \alpha} \frac{v^{\prime 2}}{v_{m}^{2}}-\frac{(1+\alpha)^{2}}{4 \alpha} \frac{v^{2}}{v_{m}^{2}}-\frac{(\alpha+1)(\alpha-1)}{2 \alpha} \frac{v v^{\prime}}{v_{m}^{2}} \cos \theta+\frac{\alpha v^{2} v^{\prime 2} \sin ^{2} \theta}{v_{m}^{2}\left(v^{2}-2 v v^{\prime} \cos \theta+v^{\prime 2}\right)}\right),
\end{aligned}
$$

where

$$
v^{(0)}=(8 / 3) \sqrt{\pi}\left(\alpha^{-1}+1\right)^{-1 / 2} v_{m} n_{2} d^{2}
$$

is the first-order effective frequency of velocity-changing collisions [24, 46], $\alpha$ is the perturber-to-active-molecule mass ratio, $d$ is the mean hard-sphere diameter of the colliding particles, and $n_{2}$ is the density number of perturbers. The relation with frequency of the velocity-changing collisions, $v_{\mathrm{VC}}$, is

$$
v_{\mathrm{VC}}=v^{(0)} / f_{D}
$$

where the $\alpha$-dependent coefficient, $f_{D}$, can be determined from Eq. (19); see Refs. [14, 24, 46]. For the self-perturbed $\mathrm{D}_{2}$, $\alpha=1, f_{D} \approx 1.01895$. In Fig. 2, panels (c) and (d), we compare the $\mathrm{HC}$ and BB kernels. It is clearly observed that the simple phenomenological HC kernel does not account for the angle and initial velocity dependencies and can differ from the real kernel by more than one order of magnitude. These unphysical properties of the HC kernel are clearly observed in experimental spectra analysis as an inability to properly reproduce the shapes of molecular hydrogen rovibrational lines in a wide range of pressures [15, 16, 42-44]. It should be emphasized that the BB kernel also correctly handles the strong mass dependence of the kernel, whereas the HC kernel does not even depend on $\alpha$. We denote the normalized operator of the velocity-changing collisions arising from the BB kernel as $\hat{M}_{\mathrm{BB}}^{f}$. The direct expression for the decomposition of $\hat{M}_{\mathrm{BB}}^{f}$ in the Burnett basis is given in Refs. [24, 46].

For the case of molecular hydrogen (and all of its isotopologues), the speed dependence of the collisional broadening, $\Gamma$, and shift, $\Delta$, is very pronounced and cannot be ignored in the experimental spectrum analysis. Therefore, the simple expression for $\Gamma_{0}$ and $\Delta_{0}$, Eq. (14), has to be replaced with its speed-dependent counterpart. Introducing the conditional probability, $f_{m_{2}}$, of having a relative speed $v_{r}$ when the active 

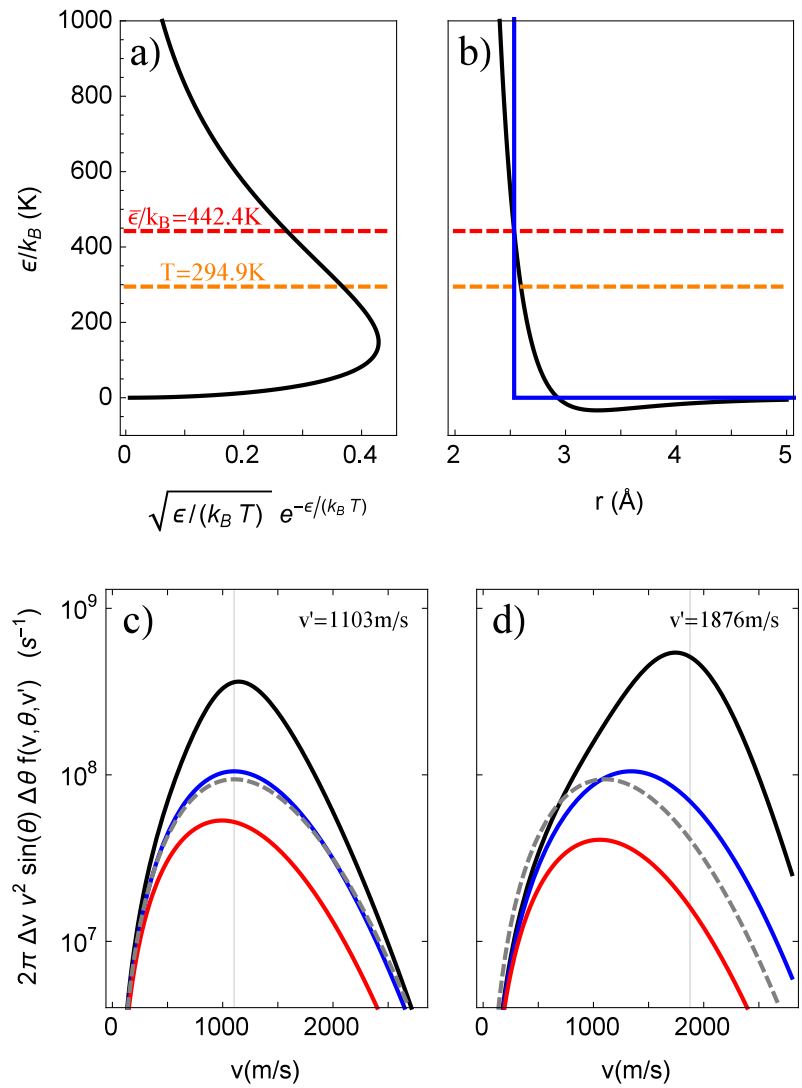

Figure 2: (a) Maxwell-Boltzmann collisional energy distribution at $T=294.9 \mathrm{~K}$. (b) Isotropic part of the $\mathrm{D}_{2}-\mathrm{D}_{2}$ potential. The blue line is the hard-sphere approximation whose diameter is set by choosing the intersection point at the mean collision energy, i.e., $\bar{\epsilon} / k_{B}=442.4 \mathrm{~K}$. (c) and (d) Collision kernel for the $\mathrm{D}_{2}-\mathrm{D}_{2}$ hard-sphere approximation at $\mathrm{T}=294.9 \mathrm{~K}$ and $\mathrm{n}_{\mathrm{H}_{2}}=1$ amg. The black, blue, and red colors correspond to scattering angles $\theta=36^{\circ}, 90^{\circ}$ and $144^{\circ}$, respectively. Charts (c) and (d) were plotted for initial speeds $v^{\prime}=1563 \mathrm{~m} / \mathrm{s}$ and $2657 \mathrm{~m} / \mathrm{s}$. For comparison, the hard-collision kernel is plotted as a gray dashed line. The $\theta$ angle element $\sin \theta \Delta \theta=\Delta(-\cos \theta)=0.2$, and the speed element $\Delta v=200 \mathrm{~m} / \mathrm{s}$.

molecule speed is $v$ at the temperature $T$, the speed dependent collisional width and shift reads [47-49]:

$$
\begin{aligned}
& \Gamma(v)+i \Delta(v)= \\
& \frac{1}{2 \pi c} \frac{p}{k_{B} T} \sum_{j_{2}} p_{j_{2}} \int d^{3} \vec{v}_{r} f_{m_{2}}\left(\vec{v}+\vec{v}_{r}\right) v_{r} \sigma_{0}^{q}\left(v_{i} j_{i} v_{f} j_{f} j_{2} ; v_{r}\right) .
\end{aligned}
$$

The integrals over the angle coordinates of $\vec{v}_{r}$ can be performed analytically [47-49]:

$$
\begin{aligned}
& \Gamma(v)+i \Delta(v)= \\
& \frac{1}{2 \pi c} \frac{p}{k_{B} T} \sum_{j_{2}} p_{j_{2}} \frac{2}{\sqrt{\pi} \bar{v}_{p} v} \int_{0}^{\infty} d v_{r} v_{r}^{2} e^{-\frac{v^{2}+v_{r}^{2}}{\bar{v}_{p}^{2}}} . \\
& \cdot \sinh \left(\frac{2 v v_{r}}{\bar{v}_{p}^{2}}\right) \sigma_{0}^{q}\left(v_{i} j_{i} v_{f} j_{f} j_{2} ; v_{r}\right),
\end{aligned}
$$

\subsection{Experimental setup}

We measured the $\mathrm{S}(2)$ line from the 2-0 band of $\mathrm{D}_{2}$ at four pressures $(247.2,471.3,743.8$ and 984.4 Torr) and at a temperature of $294.9 \mathrm{~K}$. We used a deuterium sample having a 
purity of $99.96 \%$. The spectra were collected with a frequencystabilized cavity ring-down spectrometer (FS-CRDS) linked to an optical frequency comb (OFC) [53]. The OFC was referenced to a primary time standard, the UTC(AOS) (Coordinated Universal Time from the Astro-Geodynamic Observatory in Borowiec, Poland). The length of the cavity was $74 \mathrm{~cm}$, which corresponds to the free spectral range of $204 \mathrm{MHz}$. The cavity finesse was approximately 40000. The experimental setup is described in details in Ref. [54]. The collected spectra are shown in Fig. 4; see the red dots in the top panel. To achieve high signal-to-noise ratio (8500 at the highest pressure), approximately 85 spectra were averaged at each pressure.

\subsection{Simple pressure-by-pressure fits with Voigt and hard-collision profiles}

The simplest approach to determine the unperturbed position of the line, $v_{0}$, is to fit the spectra independently, pressure by pressure, with some simple symmetric profile and use a linear extrapolation of the pressure-dependent line position, $v_{p}(p)$, to the zero-pressure limit. We tested this approach with two models: the Voigt profile (VP) and the hard-collision profile (HCP). The residuals are shown in Fig. 4, panels (a) and (b), and the retrieved values of the parameters are shown in Table 1, rows (a) and (b). For the case of the VP, the values of the line-shape parameters are completely unphysical since the VP does not account for Dicke-narrowing, which is exceptionally strong in the case of $D_{2}$. The Dicke-narrowed shape is artificially reproduced by significant diminishing of $\Gamma_{D}$ and enlarging of $\gamma_{0}$; compare $\Gamma_{D}$ and $\gamma_{0}$ retrieved with the VP with the $a b$ initio values, rows (a) and (f) in Table 1. In the case of the VP, the $\Gamma_{D}$ parameter was fitted and not constrained to the value determined from the temperature (otherwise, the shape of the VP does not fit the experimental spectra at all). The values of the line-shape parameters are more reasonable when the HCP is employed since it considers Dicke-narrowing (in this case, $\Gamma_{D}$ is fixed to the value determined from temperature). Nevertheless, the fitted parameters remain far from the real values, compare with rows (c) and (f) in Table 1, and the residuals are almost as bad as in the case of the VP; see panel (b) in Fig. 4. The shapes of the two models, the VP and the HCP, are different, but since both of them are symmetric, the pressure-dependent line positions, $v_{p}$, determined with them are almost the same; see Fig. 5. The shapes of the $\mathrm{D}_{2}$ lines are asymmetric due to collisional effects, and the degree of this asymmetry depends on the pressure (the asymmetry vanishes at low pressures and saturates at some constant pressure-independent level in the high-pressure limit). Consequently, $v_{p}$ determined with a symmetric-profile fit does not scale linearly with pressure (see the top panel in Fig. 2 in Ref. [16]), which is usually neglected in the line-shape analysis. We show this nonlinear behavior in the bottom panel of Fig. 5. The linear extrapolation of $v_{p}$ to the zero-pressure limit leads to a systematic error in $v_{0}$ at the level of $0.18 \cdot 10^{-3} \mathrm{~cm}^{-1}$ or $5 \mathrm{MHz}$; see $\delta v_{0}$ for the VP and the HCP in Table 1.

It should be noted that the residuals from the HCP, shown in panel (b) in Fig. 4, would be much larger if the multi-spectrum fitting approach would be applied and a more physical behavior would be enforced (for instance, enforcing the linear pressure dependence of at least one of the collisional parameters or fixing some of these parameters to their real values).

\subsection{Ultimate multi-spectrum fit with ab initio line-shape model}

We eliminate the above-mentioned systematic error in the determination of $v_{0}$ by employing the $a b$ initio line-shape model $\left(\mathrm{SD}_{\mathrm{ai}} \mathrm{BBP}\right.$; see Sec. 2$)$ in the analysis of our experimental spectra. To avoid numerical correlations between some parameters of the line-shape model and hence systematic errors in the retrieval of their values (in particular, in the value of $v_{0}$ ), we use the multi-spectrum fit approach, and we fix the speed dependence of the broadening and shift to the $a b$ initio values. A detailed discussion on the influence of the numerical correlations is given in Sec. 3.4. To quantify the strength of the speed dependence of the broadening and shift and to have the ability to adjust its magnitude in the fitting routines (see Sec. 3.4) while preserving the $a b$ initio shape of these functions (see Fig. 3), we introduce two parameters, $\gamma_{\mathrm{SD}}$ and $\delta_{\mathrm{SD}}$, defined by the following equations:

$$
\begin{aligned}
& \gamma(v)=\gamma_{0}+\gamma_{\mathrm{SD}} b_{\gamma}(v), \\
& \delta(v)=\delta_{0}+\delta_{\mathrm{SD}} b_{\delta}(v) .
\end{aligned}
$$

To ensure the uniqueness of the choice of the $\gamma_{\mathrm{SD}}$ and $\delta_{\mathrm{SD}}$ parameters, an additional normalization-like condition has to be implied in the dimensionless functions $b_{\gamma}(v)$ and $b_{\delta}(v)$ :

$$
\left.\frac{d}{d v} b_{\gamma}(v)\right|_{\nu=v_{m}}=\left.\frac{d}{d v} b_{\delta}(v)\right|_{\nu=v_{m}}=\frac{2}{v_{m}} .
$$

It should be noted that since $\gamma_{0}$ and $\delta_{0}$ are speed-averaged values of $\gamma(v)$ and $\delta(v)$, the average of $b_{\gamma}(v)$ and $b_{\delta}(v)$ over the Maxwell velocity distribution is zero. Direct expressions for calculating $\gamma_{\mathrm{SD}}$ and $\delta_{\mathrm{SD}}$ from ab initio $\gamma(v)$ and $\delta(v)$ functions are

$$
\begin{aligned}
& \gamma_{\mathrm{SD}}=\left.\frac{v_{m}}{2} \frac{d}{d v} \gamma(v)\right|_{v=v_{m}}, \\
& \delta_{\mathrm{SD}}=\left.\frac{v_{m}}{2} \frac{d}{d v} \delta(v)\right|_{v=v_{m}} .
\end{aligned}
$$

Our best determination of the line position and the line-shape parameters, retrieved with $\mathrm{SD}_{\mathrm{ai}} \mathrm{BBP}$, are given in row (c) in Table 1 . The broadening parameter, $\gamma_{0}$, agrees with the $a b$ initio value within approximately $5 \%$; compare with row (f) in Table 1. Much worse agreement, approximately $27 \%$, is observed for the shift parameter $\delta_{0}$. The discrepancy is caused by the PES [40] inaccuracy (the line shift is substantially more sensitive to PES imperfections than the line broadening). A new $\mathrm{H}_{2}-\mathrm{H}_{2} \mathrm{PES}$, which is under development [55], will allow us to reduce this discrepancy considerably. It has to be emphasized that for the determination of the line position, $v_{0}$, the inaccuracy of the $a b$ initio line shift, $\delta_{0}$, is not that crucial because we fit the value of this parameter to experimental spectra. The critical part of our analysis is the $a b$ initio value of $\delta_{\mathrm{SD}}$ since we keep it fixed in the multi-spectrum fit procedure. This is one of the parameters determining the asymmetry of the line (see the discussion in 


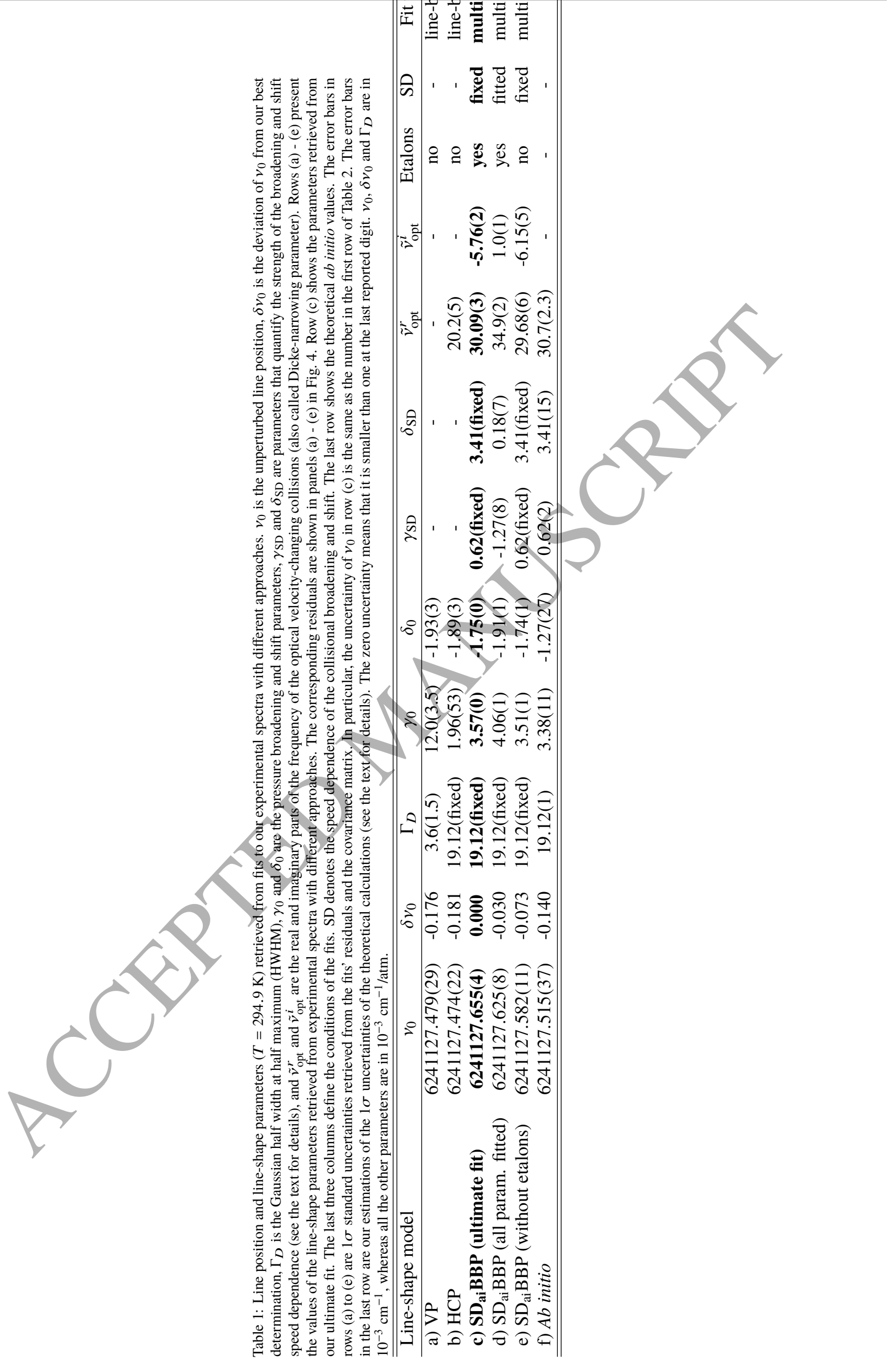


Sec. 3.4); hence, if not correctly handled, it may be responsible for the systematic error in the retrieved line position $v_{0}$. The systematic error of $a b$ initio $\delta_{\mathrm{SD}}$ is substantially smaller than the mentioned error of the $a b$ initio $\delta_{0}$. One of the reasons is that the $\delta(v)$ function (red line in Fig. 3) is almost symmetrically distributed around zero shift, and consequently, the average value, i.e., $\delta_{0}$ (the red dashed line in Fig. 3), is close to zero. The relative error of $\delta_{0}$ is as large as $27 \%$ because the reference value (i.e., the value of $\delta_{0}$ ) is close to zero. If we refer the absolute difference between the ab initio and experimental $\delta_{0}$ not to the value of $\delta_{0}$ but to the range of variability of $\delta(v)$ then the relative error is much smaller. This is why we expect a substantially smaller relative error of $\delta_{\mathrm{SD}}$, i.e., the error of the slope of the $\delta(v)$ function. To give a reliable estimation of the uncertainty of our $a b$ initio $\delta_{\mathrm{SD}}$, we take advantage of the recent measurements [56] of the temperature dependence of $\delta_{0}$ for the same line. It should be noticed that both the speed and temperature dependencies of $\delta_{0}$ have the same physical origin, i.e., the energy dependence of the spectroscopic cross-section. Therefore, one of them automatically gives information about the other. Zaborowski et al. [56] recently measured the temperature dependence $\delta_{0}(T)=$ $\delta_{0}\left(T_{\mathrm{REF}}\right)\left(T_{\mathrm{REF}} / T\right)^{n}$, with $\delta_{0}\left(T_{\mathrm{REF}}\right)=-1.69(1) \cdot 10^{-3} \mathrm{~cm}^{-1} / \mathrm{atm}$, $n=6.4(2)$ and $T_{\mathrm{REF}}=296 \mathrm{~K}$, whereas our $a b$ initio calculations gave $\delta_{0}\left(T_{\mathrm{REF}}\right)=-1.23 \cdot 10^{-3} \mathrm{~cm}^{-1} / \mathrm{atm}$ and $n=7.7$. Assuming a quadratic approximation of $\delta_{\mathrm{SD}}$ (see Appendix $\mathrm{A}$ ), the relation between $\delta_{\mathrm{SD}}$ and $n$ is given by a simple analytical formula: $\delta_{\mathrm{SD}}(T) \approx \delta_{2}(T)=\delta_{0}(T)(1-n) / 3[44,57,58]$. Using this expression, we estimated the uncertainty of our $a b$ initio $\delta_{\mathrm{SD}}$ as the difference between the $a b$ initio and experimental $\delta_{2}$, which gives an absolute uncertainty $\sigma\left(\delta_{\mathrm{SD}}\right)=0.25 \cdot 10^{-3} \mathrm{~cm}^{-1} / \mathrm{atm}$ or relative uncertainty of $7 \%$ (the value reported in Table 1 is expressed in terms of $1 \sigma$ uncertainty). To estimate the influence of this uncertainty on the determined value of the line position $v_{0}$, we repeated the multi-spectrum fit procedure while changing the fixed value of $\delta_{\mathrm{SD}}$ by a more conservative $10 \%$. The resulting uncertainty of $v_{0}$ constitutes a dominant contribution to the uncertainty budget; see row (3) in Table 2 . It has to be emphasized that a more accurate PES will allow the uncertainty of $\delta_{\mathrm{SD}}$ to be reduced considerably - we have already demonstrated this [59] for the case of He-perturbed $\mathrm{H}_{2}$, for which a highly accurate PES is already available [60]. The theoretical value of $v_{\mathrm{opt}}^{r}$ reported in Table 1 is a classical frequency of the velocitychanging collisions from the hard-sphere approximation, which is consistent (at room temperature) with the more realistic value calculated from a mass diffusion coefficient within approximately $3 \%$ [44]. A larger contribution to the uncertainty originates from the fact that $v_{\text {opt }}^{r}$ may differ from the usual frequency of the velocity-changing collisions by as much as $\gamma_{0}$. Therefore, we estimate the $1 \sigma$ uncertainty of $v_{\mathrm{opt}}^{r}$ at $2.3 \cdot 10^{-3} \mathrm{~cm}^{-1} / \mathrm{atm}$. One should note that this quantity serves here as a reference only and it does not influence the uncertainty of the $v_{0}$ determination.

The fitted line area, within its statistical uncertainty, does not exhibit any deviations from linear scaling with pressure and the intercept value is consistent with zero; hence, the influence of the pressure gauge accuracy on the $v_{0}$ determination is negligible. We estimated the influence of the temperature instability on $v_{0}$ by examining the correlation between the temperature
Table 2: Estimated contributions to the standard uncertainty budget of our experimental determination of the frequency of the $S(2)$ 2-0 transition in $\mathrm{D}_{2}, v_{0}$ $=187104300.038$ (401) MHz.

\begin{tabular}{lc}
\hline \hline Uncertainty source (type) & $\mathrm{u}\left(v_{0}\right) / \mathrm{kHz}$ \\
\hline 1) Statistics, $1 \sigma(\mathrm{A})$ & 132 \\
2) Optical frequency comb (A+B) & $<1$ \\
3) Line-shape analysis (B) & 357 \\
4) Instrumental systematic shift (B) & 47 \\
5) Relativistic asymmetry (B) & $<3$ \\
6) Pressure gauge nonlinearity (B) & $<1$ \\
7) Etalons (B) & 59 \\
8) Temperature instability (A+B) & 100 \\
Standard combined uncertainty & $\mathbf{4 0 1}$ \\
\hline \hline
\end{tabular}

and pressure. Note that for small temperature deviations the temperature dependence of the pressure shift is linear, hence instead of checking the pressure dependence of the temperature variations of the line shift we can check the pressure dependence of temperature. The difference between the mean temperature and its extrapolation to zero pressure is $0.11 \mathrm{~K}$. The temperature dependence of $\delta_{0}$, determined from our ab initio calculations and validated with experiment, is smaller than $1 \mathrm{MHz} / \mathrm{K}$ at $1 \mathrm{~atm}$ and $294.9 \mathrm{~K}$, which allows us to estimate the temperature instability contribution as $100 \mathrm{kHz}$; see Table 2 .

\subsection{Numerical correlations between the parameters of the line- shape models and influence of the etalons}

In the previous section, we noted that the main source of the uncertainty of our determination of $v_{0}$ is the uncertainty of the fixed $a b$ initio magnitude of the speed dependence (mainly, $\delta_{\mathrm{SD}}$ ). One may wonder whether this uncertainty can be reduced simply by fitting the $\gamma_{\mathrm{SD}}$ and $\delta_{\mathrm{SD}}$ parameters (together with all the other line-shape parameters) to experimental spectra. In this section, we show that this straightforward approach is very problematic and does not provide good control over the systematic errors. The reason for this is a strong numerical correlation between the line-shape parameters, i.e., several line-shape parameters have very similar influences on the shape of the molecular line, and instead of retrieving their real values, the fitting algorithm reconstructs the shape of the line in a geometrical sense by adjusting unphysical values of the line-shape parameters. For us, particularly unwanted is a correlation between the lineshape parameters responsible for the asymmetry of the line, i.e., $\delta_{\mathrm{SD}}$ and $v_{\mathrm{opt}}^{i}$, and the parameters effectively determining the position of the line, i.e., $v_{0}$ and $\delta_{0}$. To some extent, they can be decorrelated by employing the multi-spectrum fit approach, i.e., fitting the spectra at all the pressures simultaneously and constraining a proper pressure dependence of the parameters at the same time $[63,64]$. It turns out, however, that this is insufficient. In row (d) of Table 1, we show the results of the same analysis as in our ultimate fit, which can be observed in row (c), but also adjusting the parameters $\gamma_{\mathrm{SD}}$ and $\delta_{\mathrm{SD}}$. Clearly, the retrieved values of the line-shape parameters suffer from much larger systematic errors, and some of them are completely 

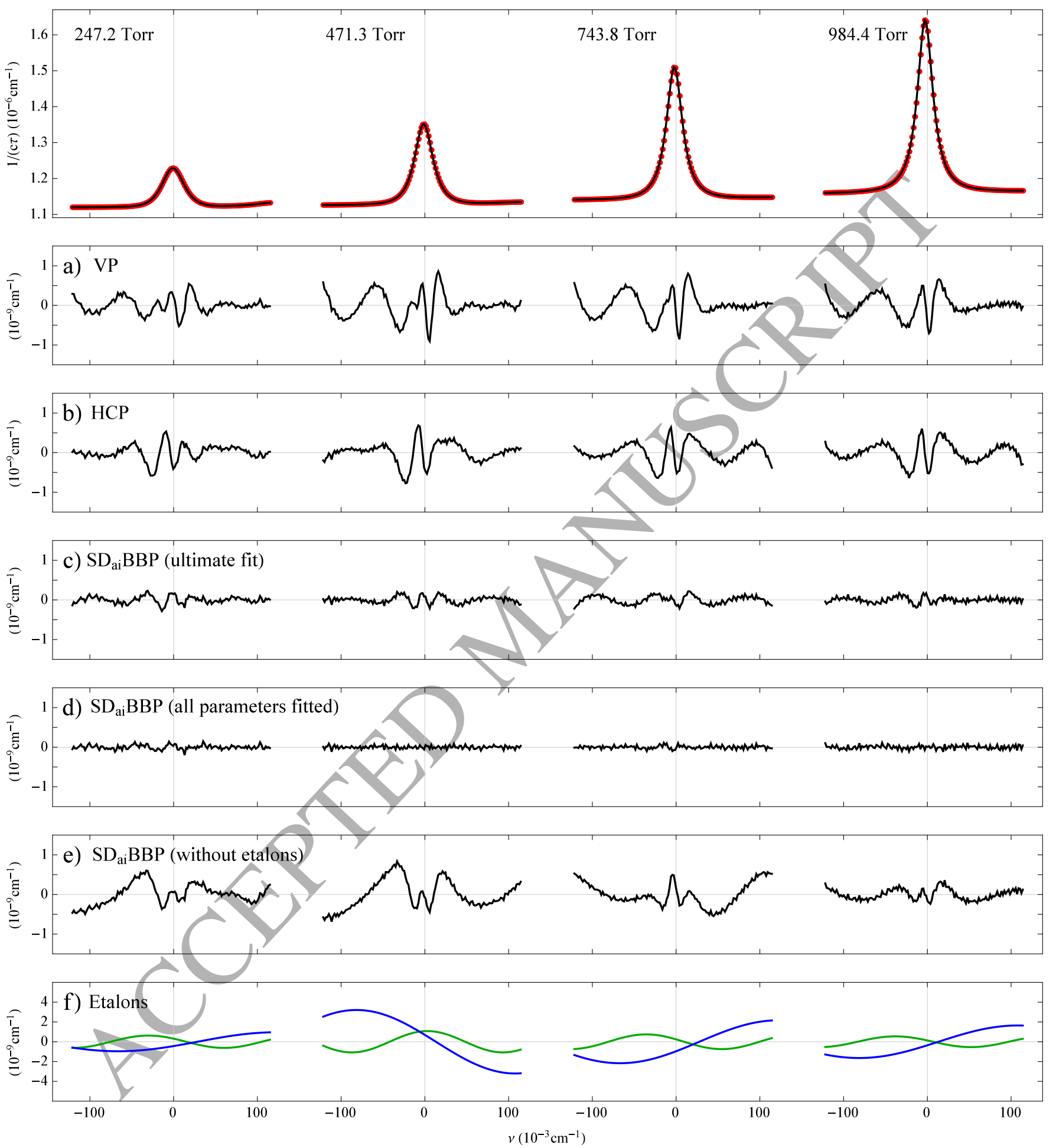

Figure 4: Rovibrational 2-0 S(2) line of $\mathrm{D}_{2}$ recorded at 247.2, 471.3, 743.8 and 984.4 torr. Panels (a) to (e) show the residuals from fits obtained with different approaches. For additional details, see Table 1. The (a)-(e) denotation is the same for the above panels and the rows in Table 1. In panel (e), we show the two etalons retrieved from our ultimate multi-spectrum fits. The blue and green lines are the etalons with fixed periods of 5.4 and $11.4 \mathrm{GHz}$, respectively, whose phases and amplitudes were fitted. 

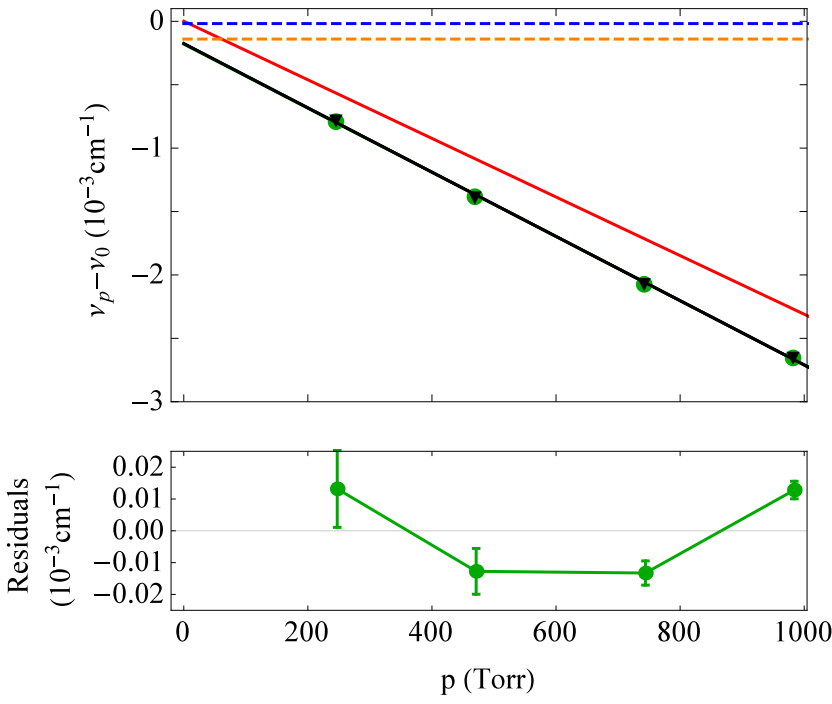

Figure 5: Pressure dependence of the collision-shifted line position, $v_{p}$, for the pressure-by-pressure fits with the Voigt profile (black triangles) and the hard-collision profile (green circles). The black and green lines are linear fits to the Voigt and hard-collision profile positions, respectively (the green line is covered by the black one). The red line is the linear pressure shift, $v_{0}+p \delta_{0}$, determined with the speed-dependent billiard-ball profile with $a b$ initio speed dependence (here, we do not have points for every pressure since the multi-spectrum fit approach was employed), where $v_{0}=6241.127655 \mathrm{~cm}^{-1}$ and $\delta_{0}=-1.756 \cdot 10^{-3} \mathrm{~cm}^{-1} / \mathrm{atm}$. The dashed blue and orange horizontal lines indicate the experimental [18] and theoretical (this work) determinations of $v_{0}$. The lower panel shows the residuals from the linear fit to the green circles.

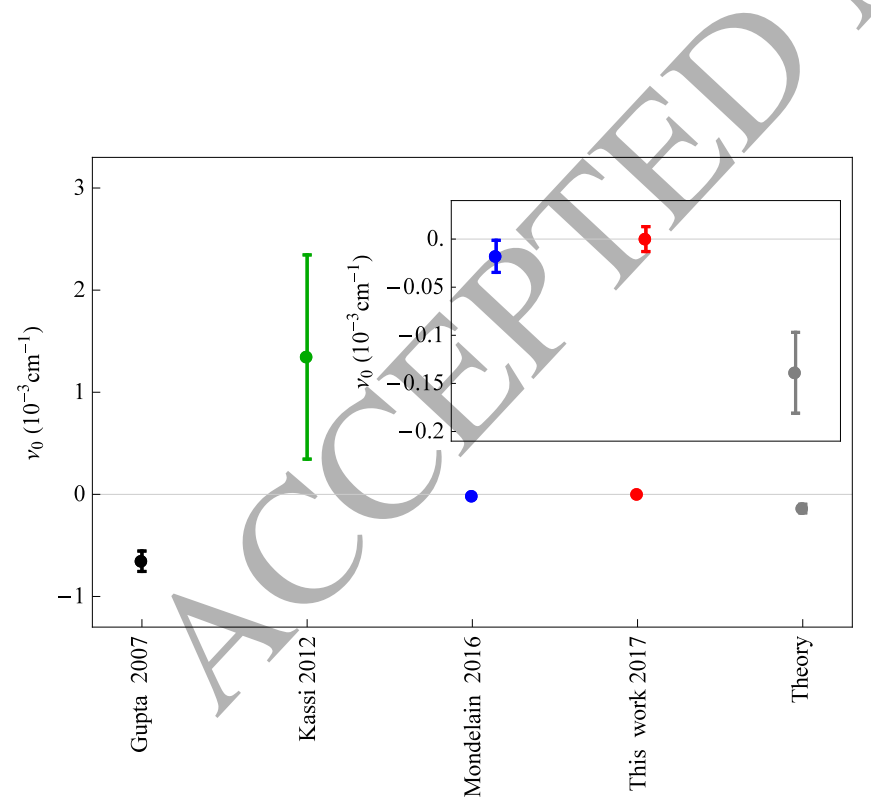

Figure 6: Comparison of our experimental (red dot) and theoretical (gray dot) determination of the energy of the $S(2)$ 2-0 line in $\mathrm{D}_{2}$ with the previous experimental results: Gupta et al. [61] (black dot), Kassi et al. [62] (green dot) and Mondelain et al. [18] (blue dot). The inset shows the last three points zoomed in on. unphysical. For instance, the $\gamma_{\mathrm{SD}}$ parameter (responsible for profile narrowing) is strongly correlated with $\gamma_{0}$ and $v_{\text {opt }}^{r}$ because all these parameters effectively determine the width of the line (none of them influence the asymmetry of the line). A comparison of their values with the results in rows (c) and (f) in Table 1 reveals the unphysical flow of the contributions between them. Larger $\gamma_{0}$ and smaller $\gamma_{\mathrm{SD}}$ increase the profile width, which is compensated by a larger value of the Dicke-narrowing parameter. The retrieved value of $\gamma_{\mathrm{SD}}$ is negative. This is completely unphysical because it implies that, for a large range of $\mathrm{D}_{2}$ speeds, the pressure broadening is negative, which does not make any physical sense. Similarly, the considerably overvalued $v_{\text {opt }}^{r}$ is certainly unphysical (the presence of the relaxations in the system makes the $v_{\mathrm{opt}}^{r}$ parameter smaller than the frequency of the velocity-changing collisions, but there is no physical mechanism that could make it larger). The same problems occur in the case of the parameters responsible for the asymmetry of the line, i.e., $\delta_{\mathrm{SD}}$ and $v_{\mathrm{opt}}^{i}$. The fitted value of $\delta_{\mathrm{SD}}$ is nearly zero, which strongly contradicts both our $a b$ initio calculations and the recent experimental temperature dependence of $\delta_{0}[56]$. The unphysical asymmetry of the line shape indispensably results in an unphysical shift in the retrieved zero-pressure line position $v_{0}$ (at the $1 \mathrm{MHz}$ level in this case). Concluding, when all the line-shape parameters are fitted, due to the strong numerical correlations, the retrieved values are completely unphysical. Within this approach, we do not have a tool to estimate the systematic error of $v_{0}$; therefore, this approach is useless from the point of view of the ultra-accurate spectroscopy of the rovibrational structure in weakly interacting molecules. Finally, one should also note that when all the lineshape parameters are fitted, the residuals are obviously smaller; see panel (d) in Fig. 4. However, despite that, the uncertainties in the retrieved parameters are considerably larger (including the $v_{0}$ parameter); see Table 1 . This is a direct manifestation of the strong numerical correlations (the residuals are smaller, but the correlations are effectively present in the covariance matrix).

In our experimental setup, two weak etalons [65] are present with periods of $5.4 \mathrm{GHz}$ and $11.4 \mathrm{GHz}$; see the green and blue lines in panel (f) in Fig. 4 (they originate from unwanted reflections from the vacuum chamber windows and are manifested in the spectra as an additional sinusoidal contribution). These periods well correspond to the distances between the highly reflective surfaces of the cavity mirrors and the surfaces of the vacuum chamber windows, which are approximately 3 and $1.5 \mathrm{~cm}$, respectively. We have been measuring the periods of the two etalons with an empty cavity in a wide frequency range. These periods are stable over the course of at least six months. To avoid introducing additional fitted parameters numerically correlated with $v_{0}$, the periods of the etalons were fixed, and only their phases and amplitudes were fitted. The strong side of our approach is that we enforce the correct physical behavior of the line shape as a function of pressure by not only employing the multi-spectrum fit but also fixing the experimentally validated [56] $a b$ initio speed dependence. This allows us to avoid situations wherein some unphysical combination of the line-shape parameters and etalons phases and amplitudes is fitted. To illustrate the necessity of employing the two etalons in our analysis, we show in panel (e) in Fig. 4 the residuals from the same fit as in our ultimate 
approach but without fitting the etalons; the retrieved values of the line-shape parameters are given in row (e) in Table 1. The fitted line-shape model was slightly perturbed to compensate the lack of the etalons in the spectrum model. This effectively leads to a systematic error in the $v_{0}$ determination at the level of $2 \mathrm{MHz}$. In our ultimate fit, row (c) in Table 1, we estimated the etalons' contribution to the uncertainty budget to be $59 \mathrm{kHz}$ by varying the fixed values of their periods by a conservative amount of $10 \%$; see row (7) in Table 2. It has to be emphasized that in future experiments aiming at kilohertz accuracy of $v_{0}$, the contribution of the etalons does not constitute a fundamental limitation because their influence can be considerably reduced in a few independent ways. For instance, the vacuum chamber windows can be covered with antireflection coatings and wedge mounted. The residual etalon structure can be better decorrelated from the line-shape structure by measuring the spectrum across a wider range (including very far wings where etalons dominates).

\subsection{Experimental determination fo the line position}

The value and uncertainty budget of the determined line position are given in Table 2. Figure 6 shows a comparison with the previous experimental determinations of $v_{0}$. Our result agrees with the value recently reported by the group from Grenoble [18], which has only slightly larger uncertainty. The dominant component of the uncertainty budget is the inaccuracy in the $a b$ initio calculations of the speed dependence of the collisional shift. It determines the asymmetry of the line; hence, it can be responsible for the systematic error in the retrieved line position $v_{0}$. The total combined standard uncertainty of the measurement is $401 \mathrm{kHz}$. Despite the twenty-times-shorter effective optical path, we achieved the same sub-megahertz level of accuracy as the previous Doppler-regime measurements [18] $(\sigma=500 \mathrm{kHz}$ in Ref. [18]).

\section{Ab initio calculations of rovibrational splitting}

In this section we describe our theoretical determination of $v_{0}$, which takes into account relativistic and QED corrections up to $\alpha^{5}$ ( $\alpha$ is the fine-structure constant). The experimental value of the energy of the $S(2)$ 2-0 transition in $\mathrm{D}_{2}$ reported here differs from our theoretical determination by $3.4 \sigma$; see Table 3. The reason for this discrepancy is not known. We suppose that it is most probably caused by underestimation of the combined relativistic nonadiabatic effects and not by the loss of numerical precision. The nonrelativistic energy is obtained here via a fully nonadiabatic approach with explicitly correlated exponential functions using octuple precision arithmetics [66]. The relativistic corrections $E^{(2)}$ and $E^{(4)}$, taken from $[67,68]$, were obtained using explicitly correlated Gaussian functions that exactly satisfy the interelectronic cusp condition, thus ensuring high numerical precision. Only the QED correction $E^{(3)}$ [69], calculated using the standard explicitly corrected Gaussian functions, exhibits some numerical uncertainty of relative order $10^{-3}$. The higher order correction $E^{(5)}$ is only estimated using the known atomic hydrogen results and assuming that it scales
Table 3: Contributions to the theoretical determination of the energy of the $\mathrm{S}(2)$ 2-0 transition in $\mathrm{D}_{2}$. $E^{(2)}, E^{(3)}, E^{(4)}$, and $E^{(5)}$ are calculated in the BornOppenheimer (BO) approximation; therefore, they contain the uncertainty due to nonadiabatic corrections, denoted by ( $)_{\text {na }}$, while the other uncertainty corresponds to approximate numerical evaluation. Lack of the specified uncertainty means that it is negligible. $E_{\mathrm{FS}}$ is the finite nuclear size correction. In the bottom part of the table, we compare the recent experimental determination [18] with our measurement. The last row is the combined value of these two experimental energies.

\begin{tabular}{lc}
\hline \hline Contribution & $\mathrm{cm}^{-1}$ \\
\hline$E^{(0)}$ (nonrelativistic) & $6241.120920(1)$ \\
$E^{(2)}\left(\alpha^{2}\right.$ relativistic) & $0.040057(20)_{\mathrm{na}}$ \\
$E^{(3)}\left(\alpha^{3} \mathrm{QED}\right)$ & $-0.03315(3)(2)_{\mathrm{na}}$ \\
$E^{(4)}\left(\alpha^{4} \mathrm{QED}\right)$ & -0.000299 \\
$E^{(5)}\left(\alpha^{5} \mathrm{QED}\right)$ & $0.000019(10)$ \\
$E_{\mathrm{FS}}$ & -0.000032 \\
Total theor. & $\mathbf{6 2 4 1 . 1 2 7 5 1 5}(\mathbf{4 2})$ \\
\hline Expt. ${ }^{a}$ & $6241.127637(17)$ \\
Expt. & $6241.127655(13)$ \\
Combined expt. & $\mathbf{6 2 4 1 . 1 2 7 6 4 7 ( 1 1 )}$ \\
\hline
\end{tabular}

$a_{\text {Ref. [18] }}$

${ }^{b}$ This work

proportionally to the square of the electron wave function at the nuclei. However, all the relativistic contributions have been calculated within the Born-Oppenheimer approximation and thus neglect the combined relativistic nonadiabatic corrections. These corrections usually scale with the ratio of the electron to the reduced nuclear mass, resulting in a $5 \times 10^{-4}$ factor. For the considered here $S(2)$ line, it gives a quite small uncertainty of $2 \times 10^{-5}$. The problem is that the leading relativistic correction $E^{(2)}$ is anomalously small; as a result, this reduced mass scaling may not be valid in this particular case. Indeed we observed in Ref. [68] similar discrepancies for other transitions in $\mathrm{H}_{2}$ and $\mathrm{D}_{2}$. In conclusion, the present discrepancy is most likely caused by the underestimated combined nonadiabatic-relativistic correction.

\section{Conclusion}

In this article we presented accurate measurements of the weak quadrupole $S(2)$ 2-0 line in self-perturbed $D_{2}$ in a pressure range from 247 - 984 Torr. We performed a detailed analysis of the collisional line-shape effects, which originates from fully quantum calculations of $\mathrm{D}_{2}-\mathrm{D}_{2}$ scattering. The velocity-changing collisions are handled with the collisional kernel based on hardsphere approximation of the potential. The experimental and theoretical pressure broadening and shift are consistent within $5 \%$ and $8 \%$, respectively (the discrepancy for the shift is referred not to the speed averaged value, which is close to zero, but to the range of variability of the speed-dependent shift). We use our high pressure measurement to determine the energy, $v_{0}$, of the $\mathrm{S}$ (2) 2-0 transition. The ab initio line-shape calculations allowed us to mitigate the expected collisional systematics reaching the $410 \mathrm{kHz}$ accuracy of $v_{0}$. We report theoretical determination of $v_{0}$ taking into account relativistic and QED corrections up to $\alpha^{5}$. Our 
estimation of the accuracy of the theoretical $v_{0}$ is $1.3 \mathrm{MHz}$. We observe $3.4 \sigma$ discrepancy between experimental and theoretical $v_{0}$ which, most likely, is caused by underestimation of the combined relativistic nonadiabatic effects. The presented method of employing the $a b$ initio line-shape modeling in determination of the energies of weak molecular transitions has a large potential for further improvements (considerable improvement can be reached by using more accurate PES or by using optical cavity with higher finesse), hence it potentially could be used for addressing the problems of the proton radius [70-72] and its mass [73] or for searching for new physics [1] with the help of very accurate calculations of the molecular levels [67].

\section{Acknowledgements}

The project is supported by the National Science Centre, Poland, Project Nos. 2015/19/D/ST2/02195, 2014/13/B/ST4/04598, DEC-2013/11/D/ST2/02663, 2015/17/B/ST2/02115 and DEC2012/05/D/ST2/01914. The research effort is part of the program of the National Laboratory FAMO in Torun, Poland. The research effort is also supported by the COST Action CM1405 MOLIM.

\section{Appendix A. Quadratic approximation of the speed depen- dence of $\gamma$ and $\delta$}

The definition of $\gamma_{\mathrm{SD}}$ and $\delta_{\mathrm{SD}}$ introduced in Sec. 3.3, namely, in Eqs. (25-29), is very convenient for practical purposes because, in the case of the widely used quadratic approximation,

$$
\begin{aligned}
& \gamma(v) \approx \gamma_{0}+\gamma_{2}\left(\frac{v^{2}}{v_{m}^{2}}-\frac{3}{2}\right), \\
& \delta(v) \approx \delta_{0}+\delta_{2}\left(\frac{v^{2}}{v_{m}^{2}}-\frac{3}{2}\right),
\end{aligned}
$$

the $\gamma_{\mathrm{SD}}$ and $\delta_{\mathrm{SD}}$ parameters are simply equal to $\gamma_{2}$ and $\delta_{2}$, respectively. Then, the physical meaning of this approximation is that the slopes of the quadratic and ab initio speed dependences are the same at the most probable speed $v_{m}$.

\section{References}

[1] Ubachs W, Koelemeij J, Eikema K, Salumbides E. Physics beyond the standard model from hydrogen spectroscopy. J Mol Spectrosc 2016;320:1 -12 .

[2] Salumbides E, Koelemeij J, Komasa J, Pachucki K, Eikema K, Ubachs W Bounds on fifth forces from precision measurements on molecules. Phys Rev D 2013;87:112008. doi:10.1103/PhysRevD.87.112008.

[3] Salumbides EJ, Schellekens AN, Gato-Rivera B, Ubachs W. Constraints on extra dimensions from precision molecular spectroscopy. New J Phys 2015; 17:033015.

[4] Liu J, Salumbides EJ, Hollenstein U, Koelemeij JCJ, Eikema KSE, Ubachs W, et al. Determination of the ionization and dissociation energies of the hydrogen molecule. J Chem Phys 2009;130:174306. doi:10.1063/1.3120443.

[5] Liu J, Sprecher D, Jungen C, Ubachs W, Merkt F. Determination of the ionization and dissociation energies of the deuterium molecule $\left(\mathrm{D}_{2}\right)$. J Chem Phys 2010;132:154301. doi:10.1063/1.3374426.

[6] Sprecher D, Liu J, Jungen C, Ubachs W, Merkt F. Communication: The ionization and dissociation energies of HD. J Chem Phys 2010;133:111102. doi:10.1063/1.3483462.
[7] Dickenson GD, Niu ML, Salumbides EJ, Komasa J, Eikema KSE, Pachucki $\mathrm{K}$, et al. Fundamental vibration of molecular hydrogen. Phys Rev Lett 2013;110:193601. doi:10.1103/PhysRevLett.110.193601.

[8] Niu M, Salumbides E, Dickenson G, Eikema K, Ubachs W. Precision spectroscopy of the rovibrational splittings in $\mathrm{H}_{2}, \mathrm{HD}$ and $\mathrm{D}_{2}$. J Mol Spectrosc 2014;300:44 - 54. doi:10.1016/j.jms.2014.03.011.

[9] Altmann RK, Galtier S, Dreissen LS, Eikema KSE. High-precision ramsey-comb spectroscopy at deep ultraviolet wavelengths. Phys Rev Lett 2016;117:173201. doi:10.1103/PhysRevLett.117.173201.

[10] Truong GW, Long DA, Cygan A, Lisak D, van Zee RD, Hodges JT. Comblinked, cavity ring-down spectroscopy for measurements of molecular transition frequencies at the kHz-level. J Chem Phys 2013;138:094201. doi:10.1063/1.4792372.

[11] Gatti D, Sala T, Gotti R, Cocola L, Poletto L, Prevedelli M, et al. Comblocked cavity ring-down spectrometer. J Chem Phys 2015;142:074201. doi:10.1063/1.4907939.

[12] Bielska K, Wójtewicz S, Morzyński P, Ablewski P, Cygan A, Bober M, et al. Absolute frequency determination of molecular transition in the doppler regime at kHz level of accuracy. J Quant Spectrosc Radiat Transfer 2017;201:156 - 160. doi:10,1016/j.jqsrt.2017.07.010.

[13] Sinclair PM, Berger JP, Michaut X, Saint-Loup R, Chaux R, Berger H, et al. Collisional broadening and shifting parameters of the Raman $Q$ branch of $\mathrm{H}_{2}$ perturbed by $\mathrm{N}_{2}$ determined from speed-dependent line profiles at high temperatures. Phys Rev A 1996;54:402-409. doi:10.1103/PhysRevA.54.402.

[14] Wcisło P, Tran H, Kassi S, Campargue A, Thibault F, Ciuryło R. Velocitychanging collisions in pure $\mathrm{H}_{2}$ and $\mathrm{H}_{2}-\mathrm{Ar}$ mixture. J Chem Phys 2014;141:074301. doi:10.1063/1.4892414.

[15] Wcisło P, Thibault F, Cybulski H, Ciuryło R. Strong competition between velocity-changing and phase- or state-changing collisions in $\mathrm{H}_{2}$ spectra perturbed by Ar. Phys Rev A 2015;91:052505. doi:10.1103/PhysRevA.91.052505.

[16] Wcisło P, Gordon IE, Cheng CF, Hu SM, Ciuryło R. Collision-induced line-shape effects limiting the accuracy in doppler-limited spectroscopy of $\mathrm{H}_{2}$. Phys Rev A 2016;93:022501. doi:10.1103/PhysRevA.93.022501.

[17] Cheng CF, Sun YR, Pan H, Wang J, Liu AW, Campargue A, et al. Electricquadrupole transition of $\mathrm{H}_{2}$ determined to $10^{-9}$ precision. Phys Rev A 2012;85:024501. doi:10.1103/PhysRevA.85.024501.

[18] Mondelain D, Kassi S, Sala T, Romanini D, Gatti D, Campargue A. Sub$\mathrm{MHz}$ accuracy measurement of the $\mathrm{S}(2) 2-0$ transition frequency of $\mathrm{D}_{2}$ by Comb-Assisted Cavity Ring Down spectroscopy. J Mol Spectrosc 2016;326:5 - 8.

[19] Nelkin M, Ghatak A. Simple binary collision model for Van Hove's $G_{S}(r, t)$. Phys Rev 1964;135:A4-A9. doi:10.1103/PhysRev.135.A4.

[20] Rautian SG, Sobelman II. Usp Fiz Nauk 1966;90:209. doi:10.1070/PU1967v009n05ABEH003212; [Sov. Phys. Usp. 1967;701:9].

[21] Galatry L. Simultaneous effect of Doppler and foreign gas broadening on spectral lines. Phys Rev 1961;122:1218-1223. doi:10.1103/PhysRev.122.1218.

[22] Blackmore R, Green S, Monchick L. Dicke narrowing of the polarized Stokes-Raman Q branch of the $\mathrm{v}=0 \rightarrow 1$ transition of $\mathrm{D}_{2}$ in He. J Chem Phys 1989;91:3846. doi:10.1063/1.457640.

[23] May AD, Liu WK, McCourt FRW, Ciuryło R, Sanchez-Fortún Stoker J, Shapiro D, et al. The impact theory of spectral line shapes: a paradigm shift. Can J Phys 2013;91:879-895. doi:10.1139/cjp-2012-0345.

[24] Ciuryło R, Shapiro DA, Drummond JR, May AD. Solving the lineshape problem with speed-dependent broadening and shifting and with Dicke narrowing. II. Application. Phys Rev A 2002;65:012502. doi:10.1103/PhysRevA.65.012502.

[25] Blackmore R. A modified Boltzmann kinetic equation for line shape functions. J Chem Phys 1987;87:791-800. doi:10.1063/1.453286.

[26] May AD. Molecular dynamics and a simplified master equation for spectral line shapes. Phys Rev A 1999;59:3495-3505. doi:10.1103/PhysRevA.59.3495.

[27] Shapiro DA, Ciuryło R, Drummond JR, May AD. Solving the lineshape problem with speed-dependent broadening and shifting and with Dicke narrowing. I. Formalism. Phys Rev A 2002;65:012501. doi:10.1103/PhysRevA.65.012501.

[28] Baranger M. Problem of overlapping lines in the theory of pressure broadening. Phys Rev 1958;111:494-504. doi:10.1103/PhysRev.111.494.

[29] Lisak D, Hodges JT, Ciuryło R. Comparison of semiclassical lineshape models to rovibrational $\mathrm{H}_{2} \mathrm{O}$ spectra measured by frequency- 
stabilized cavity ring-down spectroscopy. Phys Rev A 2006;73:012507. doi:10.1103/PhysRevA.73.012507.

[30] Chapman S, Cowling TG. The Mathematical Theory of Nonuniform Gases, Cambridge University Press, Cambridge, England, 1952;.

[31] Lindenfield MJ, Shizgal B. Matrix elements of the Boltzmann collision operator for gas mixtures. Chem Phys 1979;41:81 - 95. doi:10.1016/03010104(79)80134-2.

[32] Hess S. Kinetic theory of spectral line shapes. The transition between doppler broadening and collisional broadening. Physica 1972;61:80 - 94 . doi:10.1016/0031-8914(72)90035-3.

[33] Corey GC, McCourt FR. Dicke narrowing and collisional broadening of spectral lines in dilute molecular gases. J Chem Phys 1984;81:2318-2329. doi:10.1063/1.447930.

[34] Monchick L, Hunter LW. Diatomic-diatomic molecular collision integrals for pressure broadening and Dicke narrowing: A generalization of Hess's theory. J Chem Phys 1986;85:713-718. doi:10.1063/1.451277.

[35] Schaefer J, Monchick L. Line broadening of $\mathrm{HD}$ immersed in $\mathrm{He}$ and $\mathrm{H}_{2}$ gas. Astron Astrophys 1992;265:859-868.

[36] Demeio L, Green S, Monchick L. Effects of velocity changing collisions on line shapes of HF in Ar. J Chem Phys 1995;102:9160-9166. doi:10.1063/1.468864.

[37] Ben-Reuven A. Symmetry considerations in pressure-broadening theory. Phys Rev 1966;141:34-40. doi:10.1103/PhysRev.141.34.

[38] Ben-Reuven A. Impact broadening of microwave spectra. Phys Rev 1966;145:7-22. doi:10.1103/PhysRev.145.7.

[39] Green S. Rotational excitation in collisions between two rigid rotors: Alternate angular momentum coupling and pressure broadening of $\mathrm{HCl}$ by $\mathrm{H}_{2}$. Chem Phys Lett 1977;47:119 - 122. doi:10.1016/0009-2614(77)853207.

[40] Hinde RJ. A six-dimensional $\mathrm{H}_{2}-\mathrm{H}_{2}$ potential energy surface for bound state spectroscopy. J Chem Phys 2008;128:154308. doi:10.1063/1.2826340.

[41] Hutson JM, Green S. Molscat computer code, version 14. MOLSCAT computer code, version 14, distributed by Collaborative Computational Project No 6 of the UK Science and Engineering Research Council 1994;

[42] Tran H, Hartmann JM, Chaussard F, Gupta M. An isolated line-shape model based on the Keilson-Storer function for velocity changes. II. Molecular dynamics simulations and the $\mathrm{Q}(1)$ lines for pure $\mathrm{H}_{2}$. J Chem Phys 2009;131:154303. doi:10.1063/1.3247898.

[43] Ngo N, Lisak D, Tran H, Hartmann JM. An isolated line-shape model to go beyond the voigt profile in spectroscopic databases and radiative transfer codes. J Quant Spectrosc Radiat Transfe 2013;129:89 - 100 doi:10.1016/j.jqsrt.2013.05.034.

[44] Wcisło P, Gordon I, Tran H, Tan Y, Hu SM, Campargue A, et al. The implementation of non-voigt line profiles in the HITRAN database: $\mathrm{H}_{2}$ case study. J Quant Spectrosc Radiat Transfer 2016;177:75 - 91. doi:10.1016/j.jqsrt.2016.01.024

[45] Liao PF, Bjorkholm JE, Berman PR. Effects of velocity-changing collisions on two-photon and stepwise-absorption spectroscopic line shapes. Phys Rev A 1980;21:1927-1938. doi:10.1103/PhysRevA.21.1927.

[46] Lindenfeld MJ. Selfstructure factor of hardsphere gases for arbitrary ratio of bath to test particle masses. J Chem Phys 1980;73:5817-5829. doi: $10.1063 / 1.440066$.

[47] Ward J, Cooper J, Smith EW. Correlation effects in the theory of combined doppler and pressure broadening - I. Classical theory. J Quant Spectrosc Radiat Transfer 1974;14:555 - 590. doi:10.1016/0022-4073(74)90036-3

[48] Pickett HM. Effects of velocity averaging on the shapes of absorption lines. J Chem Phys 1980;73:6090-6094. doi:10.1063/1.440145.

[49] Pine AS. Asymmetries and correlations in speed-dependent Dickenarrowed line shapes of argon-broadened HF. J Quant Spectrosc Radiat Transfe 1999:62:397 - 423. doi:10.1016/S0022-4073(98)00112-5.

[50] Ciuryło R, Bielski A, Drummond JR, Lisak D, May AD, Pine AS, et al. Spectral Line Shapes, edited by C A Back, AIP, Melville, NY, 2002;:151

[51] Robert D, Bonamy L. Memory effects in speed-changing collisions and their consequences for spectral lineshape: I. collision regime. Eur Phys J D 1998;2:245-252. doi:10.1007/PL00021573.

[52] Wcisło P, Cygan A, Lisak D, Ciuryło R. Iterative approach to line-shape calculations based on the transport-relaxation equation. Phys Rev A 2013;88:012517. doi:10.1103/PhysRevA.88.012517.

[53] Cygan A, Wcisło P, Wójtewicz S, Masłowski P, Hodges JT, Ciuryło $\mathrm{R}$, et al. One-dimensional frequency-based spectroscopy. Opt Express 2015;23:14472-14486. doi:10.1364/OE.23.014472.

[54] Cygan A, Wójtewicz S, Kowzan G, Zaborowski M, Wcisło P, Nawrocki $\mathrm{J}$, et al. Absolute molecular transition frequencies measured by three cavity-enhanced spectroscopy techniques. J Chem Phys 2016;144:214202. doi: $10.1063 / 1.4952651$

[55] Patkowski K, private communication;

[56] Zaborowski M, et al. in preparation;.

[57] Lisak D, Cygan A, Wcisło P, Ciuryło R. Quadratic speed dependence of collisional broadening and shifting for atmospheric applications. J Quant Spectrosc Radiat Transfer 2015;151:43 - 48. doi:10.1016/j.jqsrt.2014.08.016.

[58] Ghysels M, Liu Q, Fleisher AJ, Hodges JT. A variable-temperature cavity ring-down spectrometer with application to line shape analysis of co2 spectra in the 1600nm region. Appl Phys B 2017;123:124. doi:10.1007/s00340017-6686-y.

[59] Słowiński M, et al. in preparation;.

[60] Thibault F, Patkowski K, Żuchowski PS, Józwiak H, Ciuryło R, Wcisło P. Rovibrational line-shape parameters for $\mathrm{H}_{2}$ in $\mathrm{He}$ and new $\mathrm{H}_{2}$-He potential energy surface. J Quant Spectrosc Radiat Transfer 2017;202:308 - 320. doi:10.1016/j.jqsrt.2017.08.014.

[61] Gupta M, Owano T, Baer DS, O'Keefe A. Quantitative determination of the $(2,0)$ band of deuterium in the near infrared via off-axis icos. Chem Phys Lett 2007;441:204 - 208. doi:http://dx.doi.org/10.1016/j.cplett.2007.05.031.

[62] Kassi S, Campargue A, Pachucki K, Komasa J. The absorption spectrum of d2: Ultrasensitive cavity ring down spectroscopy of the (2-0) band near $1.7 \mathrm{um}$ and accurate ab initio line list up to $24000 \mathrm{~cm}-1$. J Chem Phys 2012,136:184309. doi:10.1063/1.4707708.

[63] Benner DC, Rinsland CP, Devi VM, Smith MAH, Atkins D. A multispectrum nonlinear least squares fitting technique. J Quant Spectrosc Radiat Transfer 1995;53:705 - 721. doi:10.1016/0022-4073(95)00015-D.

[64] Pine AS, Ciuryło R. Multispectrum fits of Ar-broadened HF with a generalized asymmetric lineshape: Effects of correlation, hardness, speed dependence, and collision duration. J Mol Spectrosc 2001;208:180 - 187. doi:10.1006/jmsp.2001.8375.

[65] Cygan A, Lisak D, Wójtewicz S, Domysławska J, Hodges JT, Trawiński RS, et al. High-signal-to-noise-ratio laser technique for accurate measurements of spectral line parameters. Phys Rev A 2012;85:022508. doi:10.1103/PhysRevA.85.022508.

[66] Pachucki K, Komasa J. Nonadiabatic rotational states of the hydrogen molecule unpublished;

[67] Puchalski M, Komasa J, Czachorowski P, Pachucki K. Complete $\alpha^{6} m$ corrections to the ground state of $\mathrm{H}_{2}$. Phys Rev Lett 2016;117:263002. doi:10.1103/PhysRevLett.117.263002.

[68] Puchalski M, Komasa J, Pachucki K. Relativistic corrections for the ground electronic state of molecular hydrogen. Phys Rev A 2017;95:052506. doi:10.1103/PhysRevA.95.052506.

[69] Piszczatowski K, Łach G, Przybytek M, Komasa J, Pachucki K, Jeziorski B Theoretical determination of the dissociation energy of molecular hydrogen. J Chem Theory Comput 2009;5:3039-3048. doi:10.1021/ct900391p.

[70] Antognini A, Nez F, Schuhmann K, Amaro FD, Biraben F, Cardoso JMR, et al. Proton structure from the measurement of 2S-2P transition frequencies of muonic hydrogen. Science 2013;339:417-420. doi:10.1126/science. 1230016.

[71] Pohl R, Gilman R, Miller GA, Pachucki K. Muonic hydrogen and the proton radius puzzle. Annual Review of Nuclear and Particle Science 2013;63:175-204. doi:10.1146/annurev-nucl-102212-170627.

[72] Beyer A, Maisenbacher L, Matveev A, Pohl R, Khabarova K, Grinin A, et al. The rydberg constant and proton size from atomic hydrogen. Science 2017;358:79-85. doi:10.1126/science.aah6677.

[73] Heiße F, Köhler-Langes F, Rau S, Hou J, Junck S, Kracke A, et al. High-precision measurement of the proton's atomic mass. Phys Rev Lett 2017;119:033001. doi:10.1103/PhysRevLett.119.033001. 